\title{
Phospholipase $\mathrm{A}_{2}$ Engineering. Structural and Functional Roles of the Highly Conserved Active Site Residue Aspartate-99 ${ }^{\dagger}$
}

\author{
Kanagaraj Sekar,` Bao-Zhu Yu, ${ }^{\S}$ Joseph Rogers, ${ }^{\S}$ John Lutton," Xiaohong Liu, ${ }^{\perp}$ Xin Chen, ${ }^{\#}$ Ming-Daw Tsai, $,, \neq, \perp, \#$ \\ Mahendra K. Jain, ${ }^{*, \S}$ and Muttaiya Sundaralingam $*,+, \perp, \#$ \\ Departments of Chemistry and Biochemistry and Ohio State Biochemistry Program, The Ohio State University, \\ Columbus, Ohio 43210, and Department of Chemistry and Biochemistry, University of Delaware, Newark, Delaware 19716
}

Received July 1, 1996; Revised Manuscript Received December 27, $1996^{\otimes}$

\begin{abstract}
The aspartate-99 of secreted phospholipase $A_{2}$ (PLA2) has been proposed to be critical for the catalytic mechanism and interfacial activation of PLA2. Aspartate-99 connects the catalytic machinery (including the catalytic diad, the putative catalytic waters W5 and W6, and the calcium cofactor) to the hydrogen-bonding network. The latter involves Y52, Y73, the structural water, and the N-terminal region putatively required for the interfacial activation. A triple mutant of bovine pancreatic PLA2 with substitutions aspartate plus adjacent tyrosine residues (Y52,73F/D99N) was constructed, its X-ray structure was determined, and kinetic characteristics were analyzed. The kinetic properties of the D99N mutant constructed previously were also further analyzed. The X-ray structure of the Y52,73F/D99N mutant indicated a substantial disruption of the hydrogen-bonding network including the loss of the structural water similar to that seen in the structure of the D99N mutant published previously [Kumar, A., Sekharudu, Y. C., Ramakrishnan, B., Dupureur, C. M., Zhu, H., Tsai, M.-D., \& Sundaralingam, M. (1994) Protein Sci. 3, 2082-2088]. Kinetic analysis demonstrated that these mutants possessed considerable catalytic activity with a $k_{\text {cat }}$ value of about 5\% compared to WT. The values of the interfacial Michaelis constant were also little perturbed (ca. 4-fold lower for D99N and marginally higher for Y52,73F/D99N). The results taken together suggest that the hydrogen-bonding network is not critically important for interfacial activation. Instead, it is the chemical step that is perturbed, though only modestly, in the mutants.
\end{abstract}

Secreted phospholipases $\mathrm{A}_{2}$ (PLA2) $^{1}$ are small calciumdependent lipolytic enzymes that catalyze the stereospecific hydrolysis of the $s n-2$ ester bond of phospholipids at the interfaces (Jain et al., 1995). The activity of PLA2 on aggregated substrates is considerably higher than on monomolecularly dispersed zwitterionic substrates. This phenomenon is known as interfacial activation. The molecular basis of interfacial activation is not known, although the hydrogenbonding network (Figure 1) has been proposed to serve as a

$\dagger$ This work was supported by Research Grants GM41788 (to M.-D.T.), GM29703 (to M.K.J.), and GM49547 (to M.S.) from National Institutes of Health. The $R$-axis IIc image plate system was purchased by partial support from the Ohio Board of Regents Investment Fund. This is paper No. 17 in the series "Phospholipase $A_{2}$ Engineering" [for paper 16, see Huang et al. (1996)] and paper no. 6 in the series "Crystallography of Phospholipase $\mathrm{A}_{2}$ " [for paper 5, see Huang et al. (1996)].

$¥$ Department of Chemistry, The Ohio State University.

$\S$ Department of Chemistry and Biochemistry, University of Delaware.

"Permanent address: Department of Chemistry, Kenyon College, Gambier, OH 43022.

${ }^{\perp}$ Ohio State Biochemistry Program, The Ohio State University.

\# Department of Biochemistry, The Ohio State University.

${ }^{\otimes}$ Abstract published in Advance ACS Abstracts, February 15, 1997.

${ }^{1}$ Abbreviations: $\mathrm{CD}$, circular dichroism; $\mathrm{DC}_{7} \mathrm{PC}, 1,2$-diheptanoyl$s n$-glycero-3-phosphocholine; $\mathrm{DC}_{8} \mathrm{PM}, 1,2$-dioctanoyl-sn-glycero-3phosphomethanol; deoxy-LPC, 1-hexadecyl-1,3-dihydroxypropyl-3phosphocholine; DMPM, 1,2-dimyristoyl-sn-glycero-3-phosphomethanol; DTPM, 1,2-ditetradecyl-sn-glycero-3-phosphomethanol; EDTA, ethylenediaminetetraacetate; $\mathrm{Gdn} \cdot \mathrm{HCl}$, guanidine hydrochloride; MG14, 1-octyl-2-phosphonoheptyl-sn-glycero-3-phosphoethanolamine; MJ33, 1-hexadecyl-3-(trifluoroethyl)-rac-glycero-2-phosphomethanol; MJ72, 1-octyl-3-(trifluoroethyl)glycero-2-phosphohexanol; PLA2, phospholipase $\mathrm{A}_{2}$; WT, wild type. link between the active site and the interfacial recognition site (Verheij et al., 1981; Dijkstra et al., 1983).

The roles of Tyr-52, Tyr-73, and the N-terminal residues have been investigated by mutagenises (Dupureur et al., 1992a; Maliwal et al., 1994; Liu et al., 1995). The results showed that the hydrogen-bonding network involving the $\mathrm{N}$-terminal region and the conserved water molecule (W11) does not play a significant role in the chemical step of the catalytic cycle at the anionic interface. The crystal structure of the double mutant Y52,73F (Sekharudu et al., 1992) showed that although the loss of hydrogen bonds of the tyrosines was compensated by the increase in the hydrophobic contacts of the phenyl groups, the structural water was retained. However, in the single mutant D99N (Kumar et al., 1994), the structural water was absent.

To gain a better understanding of the role of the conserved structural water in hydrogen bonding to the catalytic triad and the active site residues, we have undertaken detailed analyses of the triple mutant Y52,73F/D99N of bovine pancreatic PLA2. The crystal structures of the triple mutant were solved in both trigonal and orthorhombic forms at 1.9 and $1.8 \AA$, respectively. Additionally, the structural role of D99 and the hydrogen-bonding network were examined by analyzing the conformational stability and the primary interfacial kinetic and equilibrium parameters. The results described in this paper show that D99 substitutions in the single and triple mutants led to only modest decreases in $k_{\text {cat }}$. The binding of PLA2 to the interface or the binding of the active site-directed ligands to the enzyme at the interface is little perturbed even though the structure of the hydrogenbonding network has been significantly disrupted. 


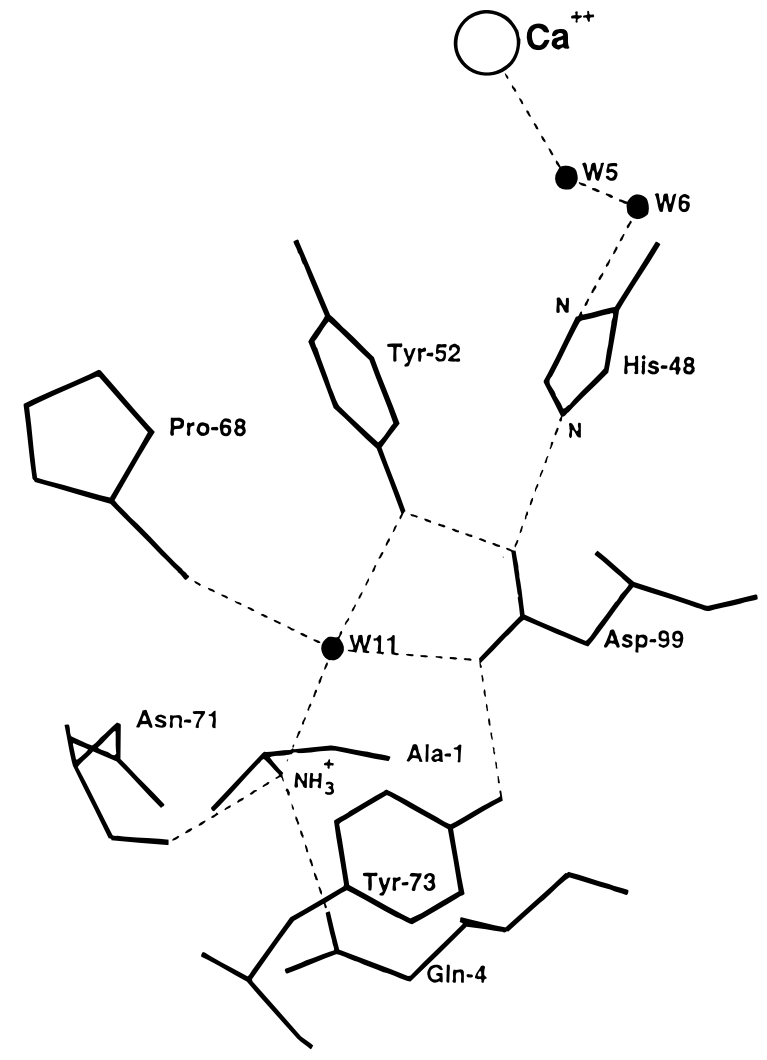

FIGURE 1: Hydrogen bonding network in the catalytic/active sites of phospholipase $\mathrm{A}_{2}$. The structural water, W11, forms a network of hydrogen bonds to the surrounding residues including Asp-99 of the active site, which in turn is involved in bonding to the other active site residue His-48. The so-called "catalytic water", W6 (Verheij et al., 1981; Dijkstra et al., 1981a,b; Scott et al., 1990a), is hydrogen bonded to the calcium-coordinated equatorial water W5. It has now been proposed that both waters W6 and W5 are involved in the mechanism of PLA2 catalysis (Rogers et al., 1996).

\section{MATERIALS AND METHODS}

Oligonucleotides were obtained from the Biochemical Instrument Center at The Ohio State University or The Midland Certified Reagent Co. (Midland, TX). Mutagenesis and sequencing kits were purchased from Amersham and United States Biochemicals, respectively. All restriction enzymes and incubation buffers were obtained from GIBCO BRL. The following lipids were synthesized as described previously: DTPM (Jain et al., 1986), DC 7 PM (Jain \& Rogers, 1989), DMPM (Jain \& Gelb, 1991), and MJ33 and MJ72 (Jain et al., 1991a). All other phospholipid substrates used in this study were purchased from Avanti Polar Lipids (Birmingham, AL). Ultrapure guanidine hydrochloride was purchased from ICN Biochemicals. The Fast Flow Sepharose-S and -Q resins (cation and anion exchangers, respectively) used for FPLC columns were purchased from Pharmacia-LKB. Other chemicals and biochemicals were of the highest quality available commercially. FPLC experiments were performed on a Pharmacia-LKB FPLC system. General protocols and strategies for the characterization of interfacial kinetic parameters are reviewed elsewhere (Jain et al., 1995), and only salient details are summarized below.

Construction and Purification of Mutant Enzymes. Sitedirected mutants were generated by using an Amersham mutagenesis kit according to the manual provided by the manufacturer. The pro-PLA2 gene from the pTO-propla2 plasmid (Deng et al., 1990) was used to construct the mutant. The double mutant Y52,73F and the single mutant D99N were constructed before (Dupureur et al., 1992; Li \& Tsai, 1993). The triple mutant Y52,73F/D 99N was constructed on the DNA template of the double mutant Y52,73F with the oligonucleotide 5'AGC GTT ACG GTT GCA GTT ACA $3^{\prime}$, where the underlined bases represent the mutated sites.

The resulting mutants were verified by direct sequencing with the dideoxy chain-termination method (Sanger et al., 1977). Recombinant PLA2 was isolated from the Escherichia coli expression host, BL21(DE3) [pLysS], carrying the pTO-propla2 plasmid as described elsewhere (Noel et al., 1991). The routine large-scale preparation of PLA2 was achieved by growth of $10 \mathrm{~L}$ of culture. The fusion proteins were activated by trypsin cleavage. As described elsewhere for the D49N mutant ( $\mathrm{Li}$ et al., 1994), it was ascertained that the residual activities of the D99N and Y52,73F/D99N mutants were not due to the presence of impurity of active enzyme as shown by the extent of hydrolysis under scooting conditions. Such a problem is expected if there is spontaneous hydrolysis of asparagine to aspartate during the folding of the protein [e.g., see Li et al. (1994)].

Preparation and Analysis of the Trigonal Form of Triple Mutant Crystals. Crystals of the triple mutant of the recombinant wild type PLA2 were obtained essentially using the same conditions described for the WT bovine enzyme (Noel et al., 1991). Small single crystals were obtained by the hanging drop method from droplets containing $5 \mu \mathrm{L}$ of the mutant protein $(15 \mathrm{mg} / \mathrm{mL}), 5 \mathrm{mM} \mathrm{CaCl} 2,50 \mathrm{mM}$ Tris buffer, $\mathrm{pH} 7.2$, and $2 \mu \mathrm{L}$ of $75 \%$ MPD. The reservoir contained 50\% MPD. Small crystals grew after 1 week and belong to the trigonal system with unit cell dimensions $a=$ $b=47.08 \AA$ and $\mathrm{c}=102.37 \AA$. X-ray diffraction data were collected on an $R$-axis II image plate (Molecular Structure Corp.) out to a resolution of $1.9 \AA$. The X-ray generator was powered at $50 \mathrm{kV}$ and $100 \mathrm{~mA}$. A total of 7346 unique reflections were collected with an $R_{\text {sym }}$ of $6.9 \%$. The data were $64 \%$ complete up to $1.9 \AA$.

Crystals of the triple mutant Y52,73F/D99N were isomorphous to the native enzyme. The starting coordinates were from the new high-resolution refinement of the trigonal form of the native enzyme (K. Sekar, unpublished results). Rigid body and positional refinements were carried out without including the mutated residues Phe-52, Phe-73, and Asn-99 using the program X-PLOR 3.0 (Brunger, 1992). This gave an $R$-value of $25.2 \%$. At this stage difference electron density maps were used to fit the three mutated residues. The entire protein model was then fitted to omit $2\left|F_{\mathrm{o}}\right|-$ $\left|F_{\mathrm{c}}\right|$ electron density maps using the molecular modeling program FRODO (Jones, 1985); after several cycles of refinement, the model was refitted wherever needed. Further refinement by simulated annealing followed by positional and individual $B$-factor refinement in the resolution range 8.0-1.9 $\AA$ gave an $R$-value of $23.3 \%$. Water molecules were located from the $\left|F_{\mathrm{o}}\right|-\left|F_{\mathrm{c}}\right|$ difference maps and included wherever they satisfied the appropriate hydrogen-bonding criteria. The $2\left|F_{\mathrm{o}}\right|-\left|F_{\mathrm{c}}\right|$ map was also used to check the quality of the water peaks. A total of 77 water molecules were found by repeated cycles of refinement. Further refinement including all the water molecules in the refinement converged the $R$ to the final value of $18.6 \%$ for 6809 reflections with $F>2 \sigma(F)$ between 8.0 and $1.9 \AA$ resolution. The final model is composed of 955 non-hydrogen protein atoms, 77 water molecules, and 1 calcium ion.

Preparation and Analysis of the Orthorhombic Form of Triple Mutant Crystals. Crystals were obtained by using the 
same conditions as for the trigonal crystals above but with a decreased concentration of 50\% MPD in the droplet. These conditions yielded orthorhombic crystals in 2 days with unit cell parameters $a=46.71 \AA, b=65.45 \AA$, and $c=38.03$ $\AA$ and isomorphous to the wild type. A total of 23993 intensity data were collected to a resolution of $1.8 \AA$ on a $R$-axis II image plate (Molecular Structure Corp.). Of these 9888 reflections are unique with an $R_{\text {sym }}$ of $7.0 \%$. The new coordinates of the high-resolution orthorhombic form of the native enzyme (K. Sekar, unpublished results) formed the starting model for the refinement of the triple mutant. Difference electron density maps, omitting the mutated residues in the refinement, were calculated after a few cycles of rigid body and positional refinement. The mutated residues were then fitted into the model using the difference electron density maps. The protein model was adjusted wherever necessary after simulated annealing and several cycles of positional and individual $B$-factor refinement. All refinements were carried out by X-PLOR 3.0 (Brunger, 1992). The molecular modeling program FRODO (Jones, 1985) was used to display the electron density maps for model fitting and adjustments. Only minor rebuilding was necessary. Water molecules were located from the difference maps, at reasonable hydrogen-bonding distances with the neighboring protein polar atoms. The final cycles of refinement of the structure including a total of 80 water molecules gave a $R$-value of $19.6 \%$ for 9413 reflections with $F>2 \sigma(F)$ in the resolution range $8.0-1.8 \AA$. The final model was comprised of 955 protein atoms, 1 calcium ion, and 80 water molecules.

$\mathrm{Gdn} \cdot \mathrm{HCl}$-Induced Denaturation and Conformational Stability. CD spectra were recorded on a JASCO J-500C spectropolarimeter using a thermostat quartz microcell and processed using DP-500/AT system (version 1.29) software. A stock solution of ca. $8.5 \mathrm{M} \mathrm{Gdn} \cdot \mathrm{HCl}$ was prepared in a buffer containing $10 \mathrm{mM}$ borate and $0.1 \mathrm{mM}$ EDTA, pH 8.0, and the exact concentration was determined by refractive index. An enzyme solution of approximately $3 \mathrm{mg} / \mathrm{mL}$ was prepared in the same buffer, and the precise concentration was measured spectrophotometrically. Samples for scanning contained $0.05 \mathrm{mg} / \mathrm{mL}$ enzyme in the borate buffer at various concentrations of $\mathrm{Gdn} \cdot \mathrm{HCl}$ at $30{ }^{\circ} \mathrm{C}$. These samples were incubated for $10 \mathrm{~min}$ before scanning five times between 250 and $200 \mathrm{~nm}$. The ellipticity at $222 \mathrm{~nm}$ was recorded and used to determine the free energy of unfolding, $G_{\mathrm{d}} \mathrm{H}_{2} \mathrm{O}$, as reported in Kumar et al. (1994).

Kinetic Analysis. All kinetic measurements were carried out on a $\mathrm{pH}$-stat titration system to monitor reaction progress as established previously (Jain et al., 1986; Berg et al., 1991). Activities toward micellar $\mathrm{DC}_{7} \mathrm{PC}$ substrates were carried out in $100 \mathrm{mM} \mathrm{NaCl}$ and $10 \mathrm{mM} \mathrm{CaCl}_{2}$, at $24{ }^{\circ} \mathrm{C}$ and $\mathrm{pH}$ 8.0. The apparent $V_{\text {max,app }}$ and apparent $K_{\mathrm{M} \text {,app }}$ for the hydrolysis of $\mathrm{DC}_{7} \mathrm{PC}$ were determined from the nonlinear regression fit of the rate versus $[\mathrm{S}]$ plot. Reaction progress curves for the hydrolysis of DMPM vesicles were recorded in $1 \mathrm{mM} \mathrm{NaCl}$ and $0.5 \mathrm{mM} \mathrm{CaCl} 2$ at $24^{\circ} \mathrm{C}$ and $\mathrm{pH} 8.0$. Analysis of these curves to obtain the turnover number, $v_{0}$, at the maximal mole fraction of the substrate at the interface $X_{\mathrm{S}}=1$ and the apparent second-order rate constant $\left(N_{\mathrm{s}} k_{\mathrm{i}}\right)$ for the hydrolysis of DMPM vesicles was conducted as described previously (Berg et al., 1991). The uncertainty in these values is $\pm 10 \%$.

Assay of PLA2 activity on DMPM vesicles in the scooting mode is facilitated considerably if the intervesicle transfer of the substrate is promoted by polymyxin B (Jain et al., 1991c). Under these conditions the microscopic steady state required for the measurement of $v_{0}$ is maintained as the observed rate is not dominated by depletion of the substrate and accumulation of the product on the enzyme-containing vesicle. Polymyxin B-induced exchange of phospholipids occurs without fusion, and it is mediated by bringing two vesicles in stable contact with each other (Cajal et al., 1996). As a consequence the apparent rate of hydrolysis would be affected if the vesicle surface is coated with the enzyme as would be the case for a catalytically inefficient enzyme. This puts a lower limit on the intrinsic rate of hydrolysis by a poor catalyst that can be measured in the scooting mode. This problem is overcome either by adding polymyxin $\mathrm{B}$ before the reaction is started with PLA2 or by using large vesicles obtained by extrusion or freeze-thaw or calciuminduced fusion (Berg et al., 1991; Jain et al., 1991b). Identical results were obtained in both cases.

The Michaelis constant for catalysis in the scooting mode at the interface $\left(K_{\mathrm{M}}{ }^{*}\right)^{2}$ was determined (Jain et al., 1995) (a) by deconvoluting the reaction progress in terms of the integrated Michaelis-Menten equation to describe catalytic turnover at the interface (Berg et al., 1991), (b) from the kinetic dissociation constant for calcium $\left[K_{\mathrm{Ca}} *(\mathrm{~S})\right]$ obtained from the dependence of $v_{0}$ on the concentration of calcium (Yu et al., 1993), or (c) from the kinetics of inhibition by active site-directed inhibitors because the mole fraction of inhibitor required for $50 \%$ inhibition, $X_{\mathrm{I}}(50)$, is related to $K_{\mathrm{I}}^{*}$ and $K_{\mathrm{M}}{ }^{*}$ (Jain et al., 1991a,d). This requires knowledge of dissociation constants for the products of hydrolysis $\left(K_{\mathrm{p}}^{*}\right)$, calcium $\left(K_{\mathrm{ca}}{ }^{*}\right)$, the ether analog of the substrate $\left(K_{\mathrm{S}}{ }^{*}\right)$, or inhibitors $\left(K_{\mathrm{I}}^{*}\right)$ bound to the enzyme at the interface of deoxy-LPC as a neutral diluent as determined by the protection described below.

Equilibrium Dissociation Constants. The equilibrium dissociation constants for active site-directed ligands $\left(K_{\mathrm{L}}{ }^{*}\right.$, where $\mathrm{L}=\mathrm{P}, \mathrm{S}, \mathrm{I}$, or $\mathrm{Ca}$ ) bound to the active site of PLA2 at the interface were determined by monitoring the rate of alkylation of His-48 by $p$-nitrophenacyl bromide as described elsewhere (Jain et al., 1991a; Yu et al., 1993), except that phenacyl bromide was used with the triple mutant. For WT and most mutants including D99N the alkylation time for the enzyme in the aqueous phase was typically $<5$ min with $p$-nitrophenacyl bromide $(2 \mathrm{mM})$; however, for the triple mutant the alkylation time was over $25 \mathrm{~min}$. On the other hand, the alkylation time for the triple mutant with phenacyl bromide was $1.7 \mathrm{~min}$ (Table 4).

The remaining PLA2 activity of each protection reaction in the presence of ligands was detected as a function of time, and the results are plotted in accordance with the ScruttonUtter equation to obtain values of the various equilibrium dissociation constants (Jain et al., 1991a; Yu et al., 1993, 1996). The uncertainty of these derived values is typically $\pm 20 \%$; however, for the triple mutant uncertainty in the

\footnotetext{
${ }^{2}$ Definition of kinetic parameters at the interface: $K_{\mathrm{Ca}}{ }^{*}$, dissociation constant for $\mathrm{Ca}^{2+}$ determined by the protection method; $K_{\mathrm{Ca}}{ }^{*}(\mathrm{~S})$, effective dissociation constant for $\mathrm{Ca}^{2+}$ under catalytic conditions at a mole fraction of 1 of the substrate; $K_{\mathrm{I}}{ }^{*}$, dissociation constant of inhibitor; $K_{\mathrm{M}} *$, Michaelis constant; $K_{\mathrm{P}}^{*}$, dissociation constant of product; $K_{\mathrm{S}}{ }^{*}$, dissociation constant of substrate; $k_{\text {cat }}$, turnover number at saturating substrate concentration; $N_{\mathrm{S}} k_{\mathrm{i}}$, apparent second-order rate constant; $v_{\mathrm{o}}$, turnover number at $X_{\mathrm{S}}=1 ; X_{\mathrm{I}}$, mole fraction of inhibitor. It should be noted that notations $K_{\mathrm{I}}^{*}, K_{\mathrm{M}}^{*}, K_{\mathrm{P}}^{*}$, and $K_{\mathrm{S}}{ }^{*}$ correspond to $K_{\mathrm{I}}, K_{\mathrm{MS}}, K_{\mathrm{P}}$, and $K_{\mathrm{S}}$, respectively, used previously (Berg et al., 1991 ).
} 

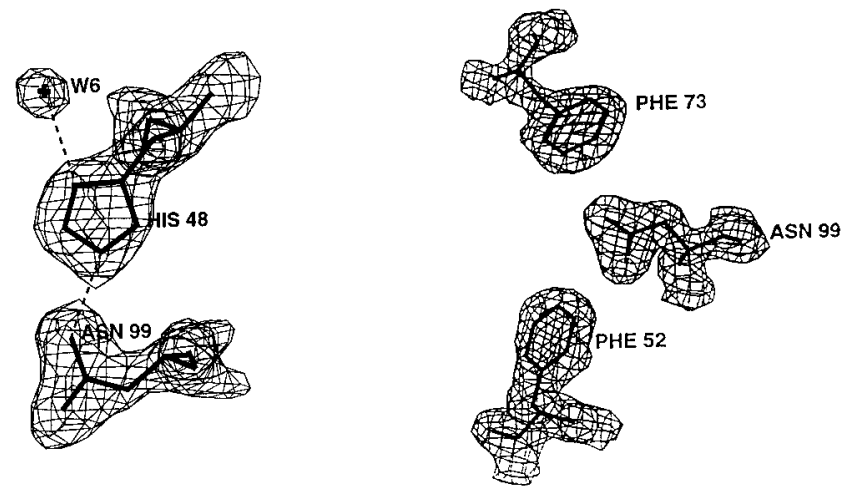

a

b

FIGURE 2: Clarity of the omit electron density map in the triple mutant, contoured at $1 \sigma$. (a) The catalytic diad, His-48 and Asn99 , and the catalytic water W6 are shown, together with the hydrogen bonding. (b) The three mutated residues, Y73F, D99N, and Y52F, which are not hydrogen bonded in both forms, are shown for the orthorhombic form.

reported values is $<50 \%$. In all cases, except one, the saturating mole fraction of the active site-directed ligand offered virtually a complete $(>97 \%)$ protection against alkylation. The saturating concentration of calcium offered only $60 \%$ protection in the $\mathrm{E}$ or $\mathrm{E}^{*}$ form of the triple mutant, although $>97 \%$ protection is seen with the ternary complexes of $\mathrm{E}^{*}$ with calcium and $\mathrm{I}, \mathrm{S}$, or $\mathrm{P}$. These measurements with the triple mutant were carried out with $3 \mathrm{mM}$ calcium compared to $0.5 \mathrm{mM}$ used for all other mutants.

Spectroscopic Measurements. All spectroscopic measurements were made in $10 \mathrm{mM}$ Tris and $0.5 \mathrm{mM} \mathrm{CaCl}_{2}$ at $\mathrm{pH}$ 8.0 and $24^{\circ} \mathrm{C}$. Unless stated otherwise, for the steady-state fluorescence measurements the excitation wavelength was $292 \mathrm{~nm}$ and the protein concentration was between 3 and $10 \mu \mathrm{M}$ with no noticeable inner filter effects. Steady-state fluorescence spectra were obtained on an SLM-Aminco AB2 or $4800 \mathrm{~S}$ spectrophotometer. The ultraviolet absorption spectra were measured with protein concentration between 20 and $35 \mu \mathrm{M}$ on a HP8452 spectrophotometer (HewlettPackard) equipped with a diode array detector. UV difference spectra and corrections for turbidity were carried out with the software package provided with the instrument.

\section{RESULTS}

X-ray Structure of the Trigonal Form of the Triple Mutant. The electron density for the mutated residues is clear in the final omit $2\left|F_{\mathrm{o}}\right|-\left|F_{\mathrm{c}}\right|$ difference map, and that of the catalytic triad is shown in Figure 2a. As usual His-48 has two hydrogen bonds, one to $\mathrm{O} \delta 1$ of Asn-99 and the other to water 6 . The electron density for the surface loop region consisting of residues $60-70$ is weak. The root mean square (rms) deviations from stereochemical standard bond lengths and bond angles are $0.015 \AA$ and $2.7^{\circ}$, respectively. The quality of the stereochemistry of the protein model was assessed using PROCHECK (Laskowski et al., 1993). All the main chain torsion angles fall within the allowed regions of the Ramachandran plot. The average estimated error in the atomic coordinates estimated using the Luzzati plot (Luzzati, 1952) is about $0.2 \AA$. All of the crystallographic parameters are summarized in Table 1.

The structural water was also found to be missing in the triple mutant as in the single D99N mutant (Kumar et al., 1994). It appears that mutating Asp-99 to Asn-99, in both
Table 1: Comparison of Selected X-ray Parameters for the Trigonal and Orthorhombic Forms of the Triple Mutant

\begin{tabular}{lll}
\hline & \multicolumn{1}{c}{ trigonal } & \multicolumn{1}{c}{ orthorhombic } \\
\hline cell $(\AA)$ & $a=b=47.08$, & $a=46.71, b=65.45$, \\
& $c=102.37$ & \multicolumn{1}{c}{$c=38.03$} \\
resolution range $(\AA)$ & $8.0-1.9$ & $8.0-1.8$ \\
space group & $P 3_{1} 21$ & $P 2{ }_{1} 2_{1}$ \\
observed reflections & 12043 & 22721 \\
unique reflections & 7346 & 9538 \\
$R_{\text {merge }}$ & 6.9 & 7.0 \\
crystallographic $R$-factor $(\%)$ & 18.6 & 19.6 \\
protein model & & \\
$\quad$ protein atoms & 955 & 955 \\
$\quad$ water molecules & 77 & 80 \\
$\quad$ bound calcium ion $\left(\mathrm{Ca}^{2+}\right)$ & 1 & 1 \\
rms deviation of structure & & \\
$\quad$ bond lengths $(\AA)$ & 0.015 & 0.011 \\
$\quad$ bond angles $(\mathrm{deg})$ & 2.7 & 1.8 \\
$\quad$ torsion angles $(\mathrm{deg})$ & 23.1 & 22.5 \\
$\quad$ impropers $(\mathrm{deg})$ & 1.2 & 1.6 \\
av temp factors of refined & & \\
structure $\left(\AA^{2}\right)$ & & 24.3 \\
$\quad$ main chain & 24.0 & 28.8 \\
$\quad$ side chain & 27.5 & 38.2 \\
$\quad$ water molecules & 34.8 & 23.5 \\
$\quad$ calcium ion & 25.0 & \\
\hline
\end{tabular}

the single and the triple mutants, is responsible for knocking out the structural water which is heavily involved in the hydrogen-bonding network. The $\mathrm{C}_{\alpha}$ atoms show an rms deviation of $0.32 \AA$ with the newly refined trigonal WT (K. Sekar, unpublished results). This suggests that the overall folding of the triple mutant is very similar to that of the WT enzyme. The main chain $\mathrm{C}_{\alpha}$ atoms of Ala-1 and the two surface loop residues Val-65 and Asp-66 show deviations of more than $1 \AA$ from the WT. Of the active site residues Ala-1, His-48, Tyr/Phe-52, Pro-68, Tyr/Phe-73, and Asp/ Asn-99 (Figure 3a), only Ala-1 shows large deviation $(>1$ $\AA$ ), while the backbone atoms of the remaining active site residues show good agreement $(0.37 \AA)$. Even though the global rms differences are small, the local differences around the conserved structural water are large. The differences in the hydrogen bonding between the triple mutant and the WT enzyme are mainly due to the absence of the structural water. Analysis of the calcium loop region indicates that the coordination around the calcium ion in the triple mutant is the same as in the trigonal form of the WT and D99N.

$X$-ray Structure of the Orthorhombic Form of the Triple Mutant. The mutated residues of the orthorhombic form are shown in the omit $2\left|F_{\mathrm{o}}\right|-\left|F_{\mathrm{c}}\right|$ electron density map (Figure $2 \mathrm{~b})$. The rms deviation of the orthorhombic mutant is 0.011 $\AA$ in bond lengths and $1.8^{\circ}$ in bond angles. Other crystallographic parameters are also summarized in Table 1 . The Ramachandran plot shows that all $(\phi, \psi)$ angles are within the acceptable regions, except for the disordered surface loop residues Leu-64, Val-65, and Asn-79 which have weak electron densities. The mean positional coordinate error of the final model is $0.2 \AA$ estimated from the Luzatti plot (Luzzati, 1952). The rms deviation of all the $\mathrm{C}_{\alpha}$ atoms of the triple mutant and the newly refined high-resolution structure of the orthorhombic form of the WT enzyme (K. Sekar, unpublished results) is $0.50 \AA$. This suggests that the folding of the triple mutant is similar to the WT. The N-terminal Ala-1, Trp-3 and the C-terminal Lys-120, Lys121 and the surface loop residues Asp-66, Tyr-69, Thr-70, and Asn-71 show more than twice the rms deviation. The rms deviation of the backbone atoms of the catalytic site residues is $0.61 \AA$ (Figure $3 b$ ) with the largest deviation of 

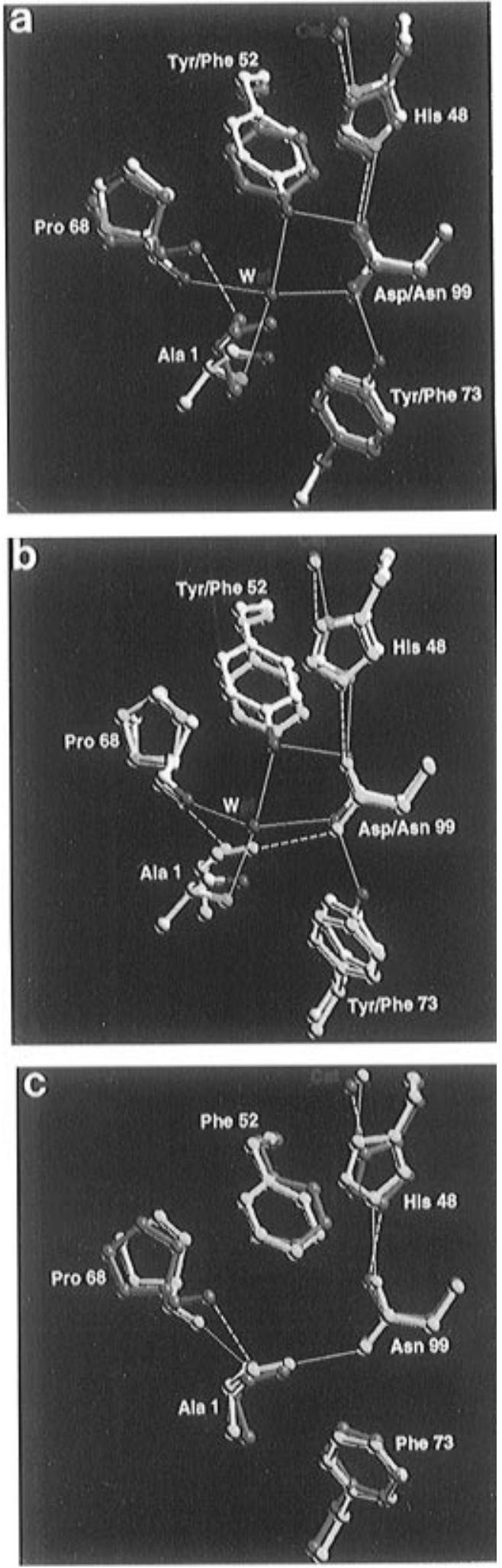

FIGURE 3: Superposition of the active site residues around the "conserved" structural water (shown here as W) and the catalytic water attached to His-48. (a) Trigonal triple mutant (purple) superposed on the trigonal WT (white). (b) Orthorhombic triple mutant (yellow) superposed on the orthorhombic WT (white). (c) Trigonal triple mutant (purple) superposed on the orthorhombic triple mutant (yellow). Notice the movement of Ala-1 in (a) and (b).

$0.9 \AA$ shown by Ala- 1 . The rms deviation reduces to 0.26 $\AA$ when Ala-1 is omitted. The remaining active site residues show only small variations. Again the structural water that stabilizes the active site conformation by a network of hydrogen bonds is absent in the orthorhombic triple mutant. Also the coordination around the calicum ion in the triple mutant is the same as in the orthorhombic form of the WT.

Comparison of the Trigonal and Orthorhombic Forms. The $\mathrm{C}_{\alpha}$ atoms of the orthorhombic and trigonal forms of the triple mutant superpose with an rms deviation of $0.88 \AA$ (Figure 4). Only the surface loop residues 63-71, with the exception of Asn-67, Pro-68, and Tyr-69, deviate more than twice the overall rms deviation. Superposition of all the backbone atoms of the six active site residues has an rms deviation of $0.5 \AA$ (Figure 3c), and Pro 68 shows the largest deviation of $0.7 \AA$. In both crystal forms of the triple mutants, the N-terminal Ala-1 has moved into the void created by the absent structural water similar to that in the single mutant D99N (Kumar et al., 1994). The amino group and carbonyl oxygen of Ala-1 form hydrogen bonds to the carbonyl oxygen of Pro- 68 and the amide nitrogen of Phe5 , respectively, to support the access route to the active site. The orthorhombic form shows one additional hydrogen bond between the carbonyl oxygen of Ala-1 and N $\delta 2$ of Asn-99, which is absent in the trigonal form.

Comparison of the Triple Mutant with the Single Mutant D99N. The single mutant D99N (Kumar et al., 1994) showed that the conserved structural water molecule was absent. We now find that this structural water is also absent in the present triple mutant structures. The electron density of the trigonal form of the triple mutant (Figure 2c) clearly shows that Ala-1 has not rotated very much; it has the same conformation as the WT ( $\psi$ is $139^{\circ}$ in the triple mutant and $167^{\circ}$ in the WT), but has moved $0.82 \AA$ toward Asn-99. In contrast, in the single mutant D99N (Kumar et al., 1994), Ala- 1 has not only moved by $1.21 \AA$ but also rotated to a $\psi$ angle of $-39^{\circ}$.

The $\mathrm{C}_{\alpha}$ atoms of the trigonal forms of the single and triple mutants can be superimposed with an rms deviation of 0.65 $\AA$. Except for Tyr-69, the remaining surface loop residues 66-71 and Asn-122 show deviations more than twice the overall deviation. The rms deviation drops to $0.39 \AA$ for the overall structure if these residues are removed. When the backbone atoms of the active site residues of the triple mutant are superimposed on those of the D99N mutant, an rms deviation of $0.95 \AA$ was obtained (Figure 5a). When the most deviating Ala-1 and Pro-68 are removed, the rms deviation reduces to $0.14 \AA$, indicating that the positions of the three mutated residues show very small perturbations.

Comparison of the Triple Mutant with the Double Mutant $Y 52,73 F$. There is good agreement between the overall structure of the trigonal triple mutant and the trigonal double mutant Y52,73F (Sekharudu et al., 1992), except for the surface loop residues Lys-113, Asp-119, and Asn-122 which deviate by more than $1 \AA$. The backbone atoms of the six active site residues deviate by $0.74 \AA$ (Figure 5B). The largest deviation of Ala- 1 and Pro-68 again proves that these residues have moved closer together to fill the void of the absent structural water. The backbone atoms of the conserved key residues Phe-52, Phe-73, and Asp/Asn-99 are virtually superimposable with an rms of $0.19 \AA$. From the above comparison studies with the trigonal native enzyme, the single mutant D99N and the double mutant Y52,73F show that only Ala- 1 and Pro- 68 have been significantly perturbed in the D99 mutants. Further, the backbone carbonyl oxygen of Ala-1 is stabilized by a hydrogen bond with the amide nitrogen of Phe-5 as in the native enzyme. 

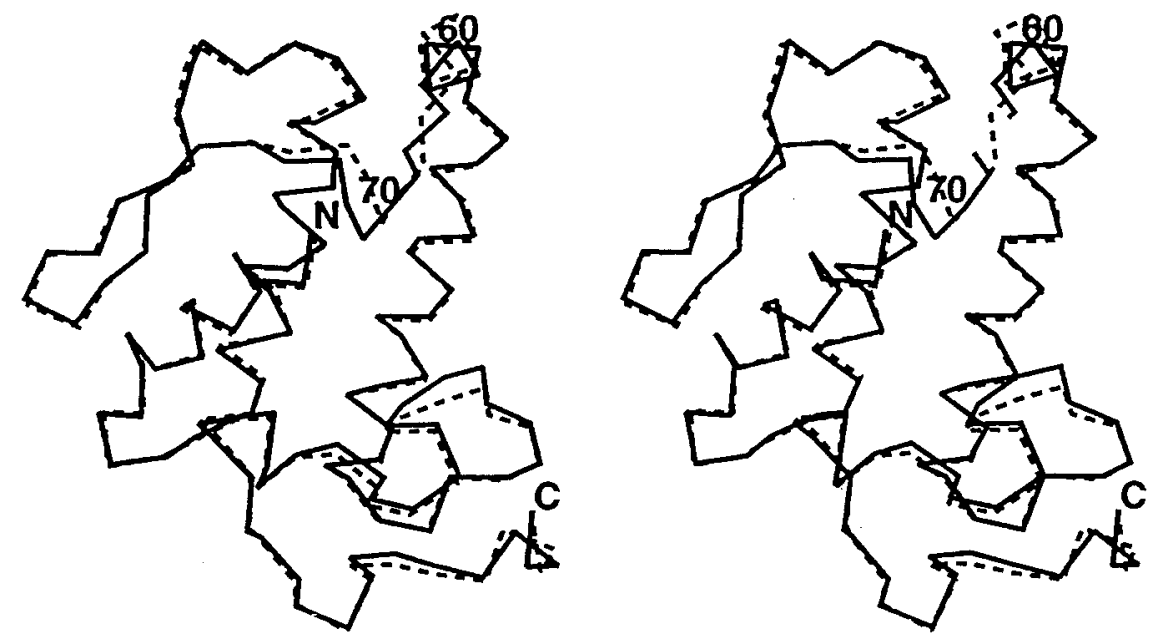

FIGURE 4: Stereoview of the $\alpha$-carbon model of the trigonal triple mutant (solid lines) superposed on the orthorhombic triple mutant (dashed lines) of the triple mutants.
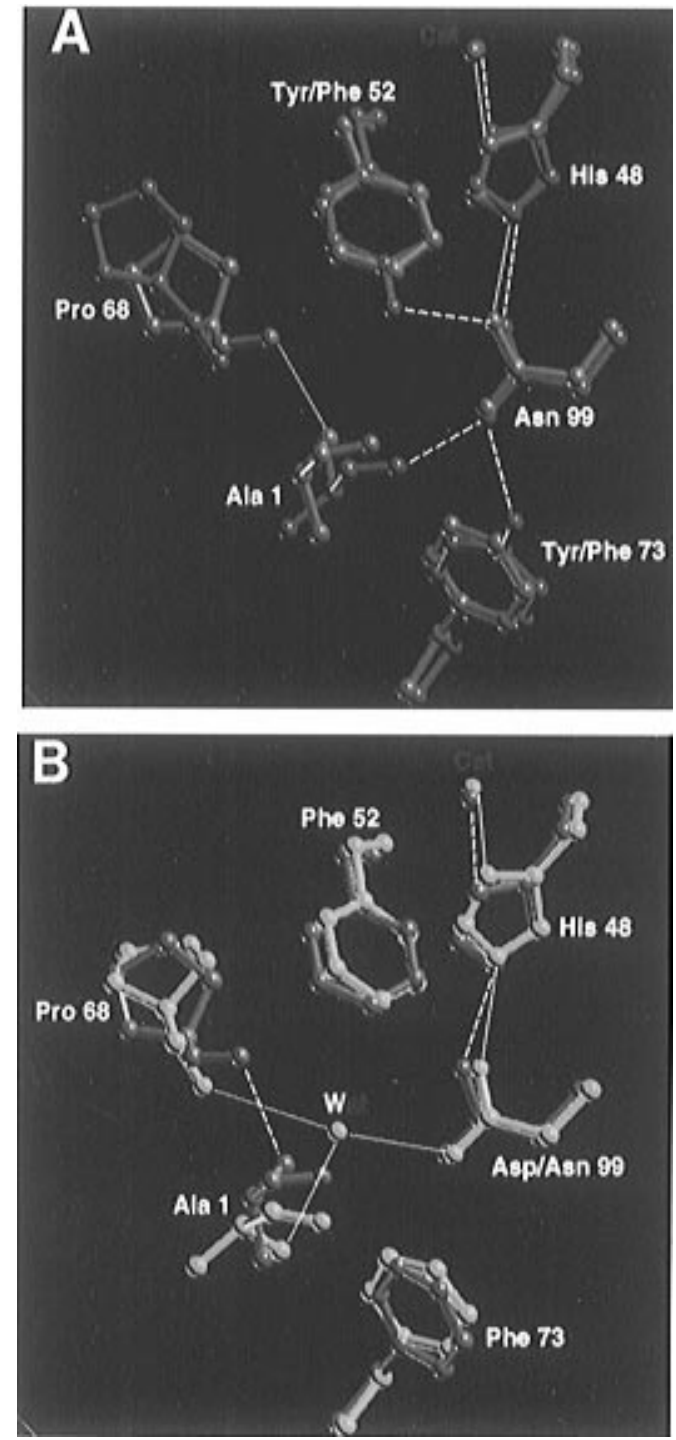

FIGURE 5: (A) Superposition of the trigonal forms of the triple mutant (purple) and the single mutant D99N (red) showing the surroundings of the missing structural water. (B) Superposition of the triple mutant (purple) on the double mutant Y52,73F (cyan) containing the structural water (shown here as W) in the trigonal forms. Notice the movement of Ala-1 and Pro-68 in both (a) and (b).

Conformational Stability of D99N and the Triple Mutant. The conformational stability of all mutants was measured
Table 2: Free Energy Changes upon Denaturation of WT and Mutants D99N and F52,73Y/D99N Induced by Gdn· $\mathrm{HCl}$

\begin{tabular}{lccc}
\hline \multicolumn{1}{c}{ enzyme } & $\Delta G_{\mathrm{d}}{ }^{\mathrm{H}_{2} \mathrm{O}}(\mathrm{kcal} / \mathrm{mol})^{a}$ & $D_{1 / 2}(\mathrm{M})$ & $m[\mathrm{kcal} /(\mathrm{mol} \cdot \mathrm{M})]$ \\
\hline $\mathrm{WT}$ & 9.5 & 6.9 & 1.48 \\
$\mathrm{D} 99 \mathrm{~N}^{b}$ & $4.7(-4.8)$ & 5.1 & 0.92 \\
$\mathrm{Y}^{2}, 73 \mathrm{~F}^{c}$ & $4.1(-5.4)$ & 5.2 & 0.79 \\
Y52,73F/D99N & $3.7(-5.8)$ & 5.0 & 0.72 \\
\hline \multicolumn{2}{c}{${ }^{a}$ Numbers in parentheses are differences between mutants and WT, } \\
i.e., $\Delta \Delta G_{\mathrm{d}} \mathrm{H}_{2} \mathrm{O} .{ }^{b}$ Data reproduced from Kumar et al. (1994). ${ }^{c}$ Data \\
reproduced from Dupureur et al. (1992a). \\
\hline
\end{tabular}

by $\mathrm{Gdn} \cdot \mathrm{HCl}$-induced denaturation, which was monitored by CD spectroscopy. Comparison of the CD spectra (200$250 \mathrm{~nm}$ ) for WT and all mutants showed only minor differences and suggested that the secondary structures are largely preserved. The denaturation curves display a behavior consistent with an apparent two-state folding mechanism (data not shown). The denaturation data were analyzed by the equation (Pace, 1986):

$$
\Delta G_{\mathrm{d}}=\Delta G_{\mathrm{d}}{ }^{\mathrm{H}_{2} \mathrm{O}}=m[\mathrm{Gdn} \cdot \mathrm{HCl}]
$$

where $\Delta G_{\mathrm{d}}$ is the Gibbs free energy change of denaturation at various concentrations of $\mathrm{Gdn} \cdot \mathrm{HCl}, \Delta G_{\mathrm{d}} \mathrm{H}_{2} \mathrm{O}$ is that at zero concentration of $\mathrm{Gdn} \cdot \mathrm{HCl}$, and $m$ is a constant related to the susceptibility of the enzyme toward denaturation by the $\mathrm{Gdn} \cdot \mathrm{HCl}$. The $\Delta G_{\mathrm{d}} \mathrm{H}_{2} \mathrm{O}$ values, the midpoint of the denaturation curve $\left(D_{1 / 2}\right)$, and the slope $(m)$ are listed in Table 2. As in the case of the single mutant D99N, the triple mutant suffers a significant loss of the free energy change of denaturation relative to the WT PLA2. The loss of stability could be related to the loss of the N-terminal structural water.

The Catalytic Turnover by D99N and the Triple Mutant Is Significant. The rate of hydrolysis of DMPM vesicles in the scooting mode was used to characterize the catalytic activity of D99 mutants. As summarized in Table 3, $v_{0}$ for the hydrolysis of DMPM vesicles by $\mathrm{D} 99 \mathrm{~N}^{3}$ was about $6 \%$ of the rate seen with the wild type. Comparable results were obtained with the short-chain analogs of the substrate $\left(\mathrm{DC}_{8^{-}}\right.$ $\mathrm{PM}$ and $\left.\mathrm{DC}_{7} \mathrm{PC}\right)$. These results are consistent with those reported for the corresponding aspartate to asparagine substitution mutants of the pig pancreatic (Kuipers et al.,

\footnotetext{
${ }^{3}$ A small portion of the kinetic data for D99N has been reported in Kumar et al. (1994).
} 
Table 3: Summary of Kinetic Data for WT and Mutants

\begin{tabular}{|c|c|c|c|c|c|c|c|}
\hline \multirow[b]{3}{*}{ enzyme } & \multirow[b]{3}{*}{$\operatorname{DMPM} v_{0}\left(\mathrm{~s}^{-1}\right)$} & \multirow[b]{3}{*}{$\mathrm{O} / \mathrm{S}$ ratio } & \multirow[b]{3}{*}{ DC8PM } & \multicolumn{4}{|c|}{ DC7PC } \\
\hline & & & & \multicolumn{3}{|c|}{ in $0.1 \mathrm{M} \mathrm{NaCl}$} & \multirow{2}{*}{$\frac{\text { in } 4 \mathrm{M} \mathrm{NaCl}}{V_{\text {max,app }}\left(\mathrm{s}^{-1}\right)}$} \\
\hline & & & & $K_{\mathrm{M}, \text { app }}(\mathrm{mM})$ & $V_{\text {max,app }}\left(\mathrm{s}^{-1}\right)$ & activation factor $^{a}$ & \\
\hline WT & 330 & 10.3 & 1000 & 3.5 & 46 & 17 & 800 \\
\hline D99N & 20 & 4.5 & 25 & 5.5 & 0.25 & 20 & 10 \\
\hline D99N/Y52,73F & 12.2 & 1.5 & 7 & 4.1 & 0.1 & 20 & 5.4 \\
\hline $\mathrm{Y} 52,73 \mathrm{~F}^{b}$ & 260 & & 2350 & & 6.9 & 16 & 280 \\
\hline
\end{tabular}

${ }^{a}$ Activation factor is defined as the ratio of $V_{\text {max,app }}$ to the rate at $1 \mathrm{mM} \mathrm{DC}_{7} \mathrm{PC}$ in $0.1 \mathrm{M} \mathrm{NaCl}$. The cmc of DC 7 PC is $1.5 \mathrm{mM}$. ${ }^{b}$ Dupureur et al. (1992a).

1990) and bee venom (Annand et al., 1996) PLA2 in other assay systems.

A critical role for D99 in the catalytic machinery of PLA2 is indicated by the fact that $v_{0}$ values for the hydrolysis of DMPM vesicles by the single D99N mutant and the triple mutant are about $5-10 \%$ of the rate obtained with the wildtype enzyme (Table 3). Also, the $\mathrm{O} / \mathrm{S}$ effect for WT is higher than that for D99N mutants (Table 3). The O/S effect is defined as the $v_{0}$ for monothio-DMPM divided by the $v_{0}$ for dithio-DMPM, as explained in Jain et al. (1992). The magnitude of the $\mathrm{O} / \mathrm{S}$ effect provides an indication as to whether the $\mathrm{C}-\mathrm{O}$ bond cleavage is a rate-limiting step in the ester hydrolysis catalyzed by PLA2. The implication of the reduced $\mathrm{O} / \mathrm{S}$ ratio for the mutants on the catalytic mechanism is elaborated in the Discussion. The following sections describe detailed characterization of the kinetic and equilibrium constants for interfacial catalysis by the D99N mutants to elaborate the basis for their lower activity without affecting the apparent interfacial activation.

The Activity of D99N Is Not Due to the Presence of the WT Enzyme as an Impurity. A possible basis for the activity in the D99N mutant is that in such preparations asparagine is spontaneously deamidated to aspartate. As developed elsewhere for the D49N mutant (Li et al., 1994) such a possibility is readily tested by monitoring catalysis in the scooting mode. Under these conditions the extent of hydrolysis of DMPM vesicles is directly related to the number of functionally active enzyme molecules in a preparation (Berg et al., 1991). For example, with $0.5 \mathrm{mM}$ $\mathrm{Ca}^{2+}$ and $0.3 \mathrm{mM}$ DMPM as sonicated vesicles, an apparently first-order reaction progress curve is seen with the WT enzyme. With D99N single and triple mutants the first-order reaction progress curve is obtained. The rate of hydrolysis under the first-order reaction progress conditions is significantly lower for the D99N mutants; the value of $N_{\mathrm{s}} K_{\mathrm{i}}$, the apparent second-order rate constant under the substrate limiting conditions, for the $\mathrm{D} 99 \mathrm{~N}$ and triple mutants was 3 and $1 \mathrm{~s}^{-1}$, respectively, as compared to $30 \mathrm{~s}^{-1}$ for the WT (Table 4). The extent of hydrolysis per D99N molecule $\left(N_{\mathrm{S}}\right)$ was the same as with the wild-type enzyme, which suggests that the residual activity of the mutant is not due to the presence of a more active enzyme as an impurity.

D99 Mutants Bind with High Affinity to Anionic Vesicles. Since D99 connects the catalytic residues to the hydrogenbonding network which may be important for interfacial binding, the interfacial binding properties of D99 mutants were investigated. The first-order reaction progress curve for the hydrolysis of small sonicated vesicles (substratelimiting conditions) showed that the bound D99N enzyme is not able to hop from one vesicle to another during the course of the reaction progress for more than 20 min because only a small fraction of the total available substrate is
Table 4: Equilibrium Binding and Catalytic Parameters for the D99 Mutants of PLA2

\begin{tabular}{|c|c|c|c|c|}
\hline parameter & D99N & WT & Y52,73F & Y52,73F/D99N \\
\hline inactivation time for $\mathrm{E}(\mathrm{min})$ & 1 & 1 & 3 & 1.7 \\
\hline$K_{\mathrm{ND}} *$ for deoxy-LPC & $>1.4$ & 1 & 1.2 & 1 \\
\hline$v_{0}\left(\mathrm{~s}^{-1}\right)\left(\right.$ at $X_{\mathrm{G}}=1$ of DMPM $)$ & 20 & 330 & 260 & 11 \\
\hline$N_{\mathrm{s}} k_{\mathrm{i}}\left(\mathrm{s}^{-1}\right)$ & 3 & 30 & 22 & 1 \\
\hline$K_{\mathrm{Ca}}(\mathrm{mM})$ for $\mathrm{ECa}$ & 0.4 & 0.5 & 0.3 & $>1$ \\
\hline$K_{\mathrm{C}} *(\mathrm{mM})$ for $\mathrm{E}^{*} \mathrm{Ca}$ & 0.51 & 0.35 & 0.15 & 0.4 \\
\hline$K_{\mathrm{Ca}} *(\mathrm{~S})($ with DMPM) $(\mathrm{mM})$ & 0.11 & 0.15 & 0.15 & 0.5 \\
\hline$K_{\mathrm{S}} *(\mathrm{E} * \mathrm{CaDTPM})$ & 0.0027 & 0.02 & 0.1 & $>0.1$ \\
\hline$K_{\mathrm{S}} *(\mathrm{E} * \mathrm{CaDTPC})$ & 0.006 & $>0.1$ & & \\
\hline$K_{\mathrm{P}}^{*}($ DMPM products $)$ & 0.009 & 0.025 & 0.024 & 0.08 \\
\hline$K_{\mathrm{I}}^{*}\left(\mathrm{E}^{*} \mathrm{MJ} 33\right)$ & 0.005 & 0.01 & 0.02 & 0.01 \\
\hline$K_{\mathrm{I}}(\mathrm{mM})$ for $\mathrm{MG} 14$ & 0.026 & 0.050 & & \\
\hline$K_{\mathrm{I}}(\mathrm{mM})$ for $\mathrm{MJ} 72$ & 0.080 & 0.120 & & \\
\hline$X_{I}(50)$ for MJ33 & 0.04 & 0.025 & 0.05 & 0.014 \\
\hline$K_{\mathrm{M}} *(\mathrm{DMPM})$ from $K_{\mathrm{I}} *$ & 0.14 & 0.65 & 0.6 & $>1$ \\
\hline$K_{\mathrm{M}} *(\mathrm{DMPM})$ from $K_{\mathrm{Ca}} *(\mathrm{~S})$ & 0.2 & 0.7 & 1 & $>1$ \\
\hline$K_{\mathrm{M}}(*$ DMPM $)$ from $v_{0} / N_{\mathrm{s}} K_{\mathrm{i}}$ & 0.07 & 0.4 & 0.67 & 1 \\
\hline$k_{\text {cat }}\left(\mathrm{s}^{-1}\right)$ & 23 & 500 & 500 & 20 \\
\hline
\end{tabular}

hydrolyzed under these conditions (Jain et al., 1986a; Berg et al., 1991). This observation alone rules out the possibility that the D99 mutants have a significantly lower affinity for the DMPM interface. This conclusion is further supported by the fact that the value of $v_{0}$ for the D99N mutant does not change with the increasing bulk concentration of DMPM vesicles, and the mutant bound to DTPM vesicles does not become available for the hydrolysis of DMPM vesicles added afterward. This is because under these conditions the catalytic turnover occurs in the highly processive scooting mode and the enzyme does not leave the interface (Jain et al., 1986, 1995). These results suggest that the binding of D99N to the anionic interface is of high affinity, similar to that of the wild-type enzyme, and that the effect of mutation is only on the steps of the catalytic turnover cycle at the interface.

Evidence for the high-affinity binding of the D99N mutant to the DMPM interface was also obtained by direct binding studies where the change in the intrinsic fluorescence of Trp-3 (Jain et al., 1986b) or the resonance energy transfer to a dansyl fluorophore acceptor located in the DTPM interface was monitored (Jain \& Vaz, 1987). This highaffinity binding of D99N to DTPM vesicles is indicated by the binding isotherms shown in Figure 6. Under these conditions the binding isotherms are steep and linear at low lipid concentrations, and the number of DTPM molecules required for the binding of each enzyme molecule is about 35 as is the case with the WT (Dupureur et al., 1992a). Evidence for a similar high-affinity binding of the D99N mutants is also indicated by resonance energy transfer from the Trp-3 donor on PLA2 to the dansyl acceptor incorporated in DTPM vesicles (results not shown). These results show that WT as well as the D99 mutants have virtually the same affinity for vesicles of anionic phospholipids. This is an 


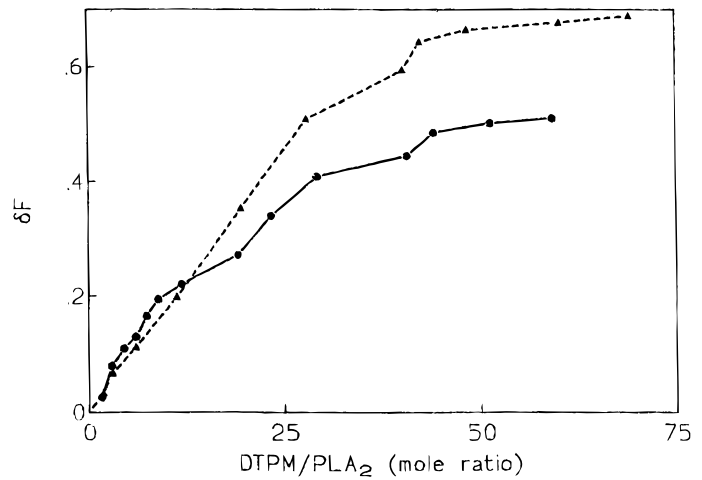

FIGURE 6: Change in the fluorescence emission intensity of (circles) D99N and (triangles) WT at $333 \mathrm{~nm}$ (excitation $292 \mathrm{~nm}$ ) with increasing mole ratio of DTPM.

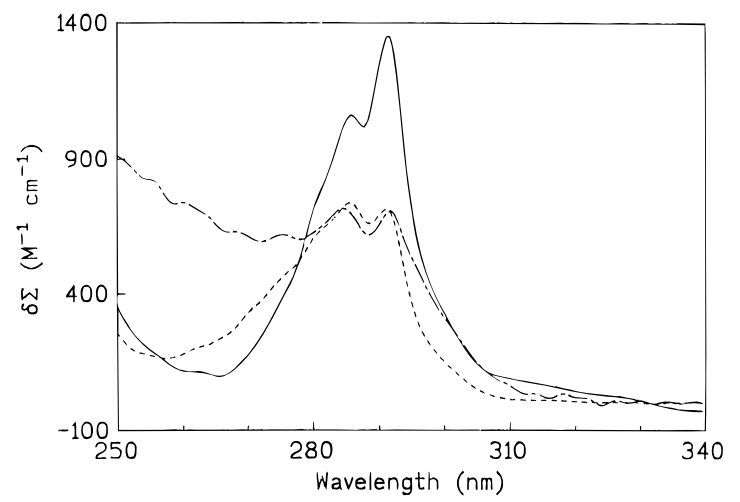

FIGURE 7: Change in the absorption spectra on the binding of MJ33 (0.05 mole fraction) to (solid line) D99N, (dashed line) D99A, and (dot-dashed line) Y52,73F/D99N PLA2.

expected result if D99 is not a residue of the interfacial recognition region of PLA2 and if the hydrogen-bonding network involving Ala-1 is not critical for interfacial binding at the anionic interface. The possibility that the H-bond network is restored in the $\mathrm{E}^{*}$ or $\mathrm{E}^{*} \mathrm{~L}$ form of the $\mathrm{D} 99 \mathrm{~N}$ mutants is discounted on the basis of the results with the des-Ala-1 mutant, which shows only a modest decrease in activity (Maliwal et al., 1994).

The Affinity for the Active Site-Directed Ligands Is Modestly Perturbed by the D99 Substitution. The origin of the effect of the D99 substitution on the steps of the interfacial catalytic turnover cycle in the scooting mode was characterized in terms of the primary kinetic and equilibrium parameters for the D99N mutant. As summarized in Table 4 , the equilibrium dissociation constants for several active site-directed ligands (calcium, inhibitors, substrate, and products) for $\mathrm{Y} 52,73 \mathrm{~F}$ and D99N mutants are comparable to those for WT. The $K_{\mathrm{P}}{ }^{*}$ and $K_{\mathrm{S}} *$ (for DTPM and DTPC) values are modestly lower for D99N and modestly higher for the triple mutant than those for the wild-type PLA2. On the other hand, the apparent affinity for the neutral diluent was very low and virtually the same as for the wild type $\left(K_{\mathrm{ND}} *>\right.$ mole fraction 1$)$ as shown in Table 4.

As shown in Figure 7, the UV spectral changes associated with the binding of MJ33 to D99N bound to the zwitterionic interface are virtually the same as those seen with WT enzyme (Dupureur et al., 1992a). The change in the absorbance at 292 depends on the mole fraction of MJ33, and the $K_{\mathrm{I}}^{*}$ obtained by the spectroscopic method is comparable to that obtained by the protection method $(0.005$ versus 0.01 mole fraction for the WT). Binding of an active site-directed ligand to PLA2 at the interface also causes an increase in the fluorescence emission intensity, and similar changes were observed with other D99 mutants. Collectively, these results suggest that the role of the D99$\mathrm{H} 48$ diad, if any, in the binding of the substrate, product, and transition state mimics is at best modestly altered in the D99N mutant and the ligand binding requires calcium in both cases.

Since the conformation of the N-terminal region of D99N mutants is appreciably altered, these spectroscopic changes in the absorption of Trp-3 imply that the binding of an active site-directed ligand to PLA2 does not require an integrity of the H-bonding network that connects the $\mathrm{N}$-terminus to the D99-H48 couple. It may also be remarked here that the changes in the absorption spectrum of D99A or D99N/ Y52,73F on the binding of MJ33 are appreciably smaller than those seen with D99N (Figure 7). We have not investigated the origin of such spectral differences in detail. However, such changes are consistent with an observed difference in the environment of Trp-3 in these mutants in the aqueous phase, probably caused by a large shift in the position of Ala-1 in the crystal structure of the D99 mutants.

$k_{\text {cat }}$ Is Altered in the D99N Mutant. The equilibrium binding and kinetic parameters summarized in Table 4 show that the only significant effect of D99N mutation is on the chemical steps of the catalytic cycle. As is the case with the wild type enzyme, the binding of all the active sitedirected ligands to the mutants required calcium as an obligatory cofactor. The $K_{\mathrm{Ca}}$ and $K_{\mathrm{Ca}}{ }^{*}$ values of D99N were marginally higher than those for the wild-type enzyme, and the $K_{\mathrm{Ca}}$ for the triple mutant is significantly higher. As expected (Yu et al., 1993) the value of $K_{\mathrm{Ca}}{ }^{*}(\mathrm{~S})$, obtained from the calcium dependence of the steady-state rate of hydrolysis of DMPM vesicles, $v_{0}$, for D99N is significantly lower than the $K_{\mathrm{Ca}}{ }^{*}$ values. As also summarized in Table 4, the value of $K_{\mathrm{M}} *$ (0.2 mole fraction) obtained from the value of $K_{\mathrm{Ca}}{ }^{*}(\mathrm{~S})$ is consistent with the values obtained by two other independent methods, i.e., from the integrated reaction progress curve and the kinetics of inhibition by active site-directed inhibitors. On the basis of the values of $v_{0}$ and $N_{\mathrm{s}} k_{\mathrm{i}}$, which were obtained from the reaction progress curves, $K_{\mathrm{M}} *$ was 0.14 mole fraction. The average $K_{\mathrm{M}} *$ for D99N based on a variety of methods was 0.2 mole fraction, which is lower than the value of 0.65 mole fraction for the wild-type enzyme.

From the values of $v_{0}$ and $K_{\mathrm{M}} *$ the rate of catalytic turnover $k_{\text {cat }}=k_{2}=23 \mathrm{~s}^{-1}$ for D99N is significantly lower than the value of $500 \mathrm{~s}^{-1}$ for the wild-type enzyme. The $k_{\text {cat }}$ for the D99N mutants, compared to the $0.1 \mathrm{~s}^{-1}$ residual activity of D99A, is surprising. This would imply that, like Asp-99, the asparagine in D99N can also play an important role in the catalytic turnover by PLA2. Similarly, the $k_{\text {cat }}$ of $20 \mathrm{~s}^{-1}$ observed for the triple mutant indicates that even loss of the majority of the hydrogen-bonding network does not eliminate the catalytic activity of the enzyme.

The $K_{\mathrm{M}} *$ values show that the difference between the $v_{0}$ values for WT and D99N mutants is due to a change in $k_{\text {cat }}$; that is, the value for $\mathrm{D} 99 \mathrm{~N}$ is about $8 \%$ of the value for the WT. These results suggest that the 3-fold decrease in the value of $K_{\mathrm{M}}{ }^{*}$ [ $\left.=\left(k_{2}+k_{-1}\right) / k_{1}\right]$ for D99N is probably not exclusively due to a decrease in $k_{2}$. A modest change in $k_{1}$ and $k_{-1}$ is indicated by the fact that the binding affinity for the substrate analogs $\left(K_{\mathrm{s}}^{*}\right)$, the products of hydrolysis $\left(K_{\mathrm{p}}{ }^{*}\right)$, and the active site-directed ligands from the ground state complex with D99N differs by a factor of about 3 when 


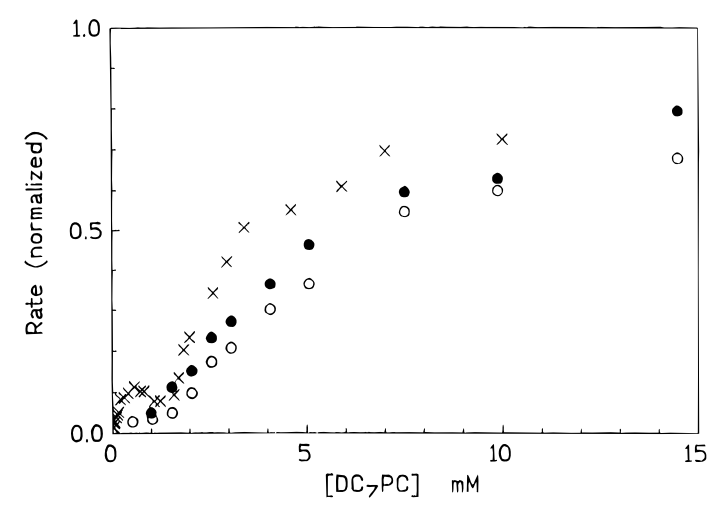

FIGURE 8: Dependence of the (normalized to $V_{\text {max,app }}$ ) initial rate of hydrolysis by (open circles) D99N, (closed circles) Y52,73F/ D99N, and (crosses) WT PLA2 of $\mathrm{DC}_{7} \mathrm{PC}$ as a function of the substrate concentration.

compared to WT. On the basis of the kinetic studies with the deletion mutants (Maliwal et al., 1994) and the crystal structure of the D99N mutant, it may now be suggested that the $\mathrm{N}$-terminal segment plays a modest role in the equilibrium binding of the active site-directed ligands to form the $\mathrm{E}^{*} \mathrm{~L}$ complex from $\mathrm{E}^{*}$.

The Apparent Interfacial Activation Is Not Altered in the D99 Mutant. An anomalous increase in the rate of hydrolysis of short-chain phosphatidylcholines at their critical micelle concentration is one of the unique features of interfacial catalysis by PLA2 (de Haas et al., 1971). As shown in Figure 8 , the rate of hydrolysis of $\mathrm{DC}_{7} \mathrm{PC}$ increases with the substrate concentration above $1.5 \mathrm{mM}$, i.e., at the $\mathrm{cmc}$ of $\mathrm{DC}_{7} \mathrm{PC}$. From the bulk substrate concentration (above $\mathrm{cmc}$ ) dependence of the rate, it is possible to obtain values of $K_{\mathrm{M} \text {,app }}$ and $V_{\text {max,app. }}$. As summarized in Table 3, although the absolute rates of hydrolysis are very different for these mutants, virtually the same rate enhancement (activation factor) above the cmc is observed with WT and the D99N and Y52,73F/D99N mutants. These results show that the D99 mutants exhibit apparent interfacial activation of the same magnitude expressed as the activation factor.

The Effect of High $\mathrm{NaCl}$ Concentration Is Not Altered in D99 Mutants. Apparent activation of the rate of hydrolysis at saturating concentrations of $\mathrm{DC}_{7} \mathrm{PC}$ is seen at high $\mathrm{NaCl}$ concentrations. As summarized in Table 3, a 40-50-fold increase in the rate of hydrolysis is seen with the various mutants compared to a 20 -fold increase seen with WT. These results show that the salt effect is not influenced by mutation appreciably, which is consistent with the fact that the increase in rate is primarily due to induction of negative charge which promotes binding of the enzyme to the interface (Jain et al., 1997). This interpretation is also consistent with the fact that the binding of the mutants to the anionic interface is comparable to that of WT. It may be recalled that the salt-induced activation is approximately 150 -fold for the mutant in which residues 115-123 are deleted (Huang et al., 1996).

The $K_{S}$ Type of Interfacial Activation Is Not Altered in $D 99 N$. Deoxy-LPC is an effective neutral diluent for the D99N and triple mutants as the inactivation time for the enzyme by alkylating agents is not altered when the enzyme is bound to the interface. As summarized in Table 5, the apparent affinity $\left(K_{\mathrm{d}}\right)$ of the free enzymes for the zwitterionic interface of the neutral diluent is about $7 \mathrm{mM}$; however, the affinity for the enzyme complexes with an active site-directed ligand $\left(K_{\mathrm{d}}^{\mathrm{I}}\right)$, such as MJ33, is $0.2 \mathrm{mM}$. A similar change is
Table 5: Spectroscopic Changes ${ }^{a}$ Associated with the Binding of Bovine PLA2 Mutants and Derived Parameters

\begin{tabular}{|c|c|c|c|c|c|}
\hline mutant & $\begin{array}{c}\% \delta F \\
\left(\mathrm{E} \rightarrow \mathrm{E}^{*}\right)\end{array}$ & $\begin{array}{c}\% \delta F \\
(\mathrm{E} \rightarrow \\
\left.\mathrm{E}^{*} \mathrm{MJ} 33\right)\end{array}$ & $\begin{array}{c}\delta \epsilon \\
\left(\mathrm{M}^{-1} \mathrm{~cm}^{-1}\right) \\
\left(\mathrm{E}^{*} \rightarrow \mathrm{E}^{*} \mathrm{MJ} 33\right)\end{array}$ & $\begin{array}{c}K_{\mathrm{d}} \\
(\mathrm{mM})\end{array}$ & $\begin{array}{c}K_{\mathrm{d}}^{1} \\
(\mathrm{mM})\end{array}$ \\
\hline WT & 17 & 57 & 1800 & 7 & 0.2 \\
\hline $\mathrm{H} 48 \mathrm{~A}$ & 17 & 69 & 2200 & 1.4 & 0.11 \\
\hline $\mathrm{H} 48 \mathrm{~N}$ & 16 & 60 & 2750 & & \\
\hline H48Q & 13 & 48 & 2700 & 1.5 & 0.06 \\
\hline Y52,73F & 17 & 40 & 2400 & 6 & 0.1 \\
\hline D99N & 18 & 34 & 1400 & 7 & 0.35 \\
\hline D99N/Y52,73F & 7 & 18 & 700 & 7 & 0.3 \\
\hline
\end{tabular}

${ }^{a}$ These changes were measured in $3 \mathrm{mM}$ deoxy-LPC. For fluorescence change saturating mole fractions of MJ33 were added, whereas $\delta \epsilon$ values were measured at 0.065 mole fraction of MJ33. See Jain et al. (1993) for detailed protocols.

seen with several other mutants (Table 5) including the D99 mutants. On the basis of the thermodynamic arguments developed elsewhere (Jain et al., 1993) the 30-70-fold increase in the affinity of the E•MJ33 form of PLA2 (WT as well as the D99N mutant) for the interface is a manifestation of the $K_{\mathrm{S}}$ type of allosteric interfacial activation, where the affinity of the enzyme at the interface for the substrate is considerably larger than that for the enzyme in the aqueous phase. This is consistent with the $K_{\mathrm{I}}$ values for D99N and WT compared in Table 4 for the short-chain inhibitors MJ72 and MG14. In summary, mutation of D99 does not alter the interfacial allosteric effect.

\section{DISCUSSION}

Aspartate-99 appears to be critically involved in a number of aspects of the structure and function of PLA2. While the X-ray structure and conformational stability of D99 mutants show significant local perturbation, detailed kinetic analysis of PLA2 functions provides evidence for a more specific role of D99 in the chemical step of the catalytic cycle.

The Active Site in D99N Mutants Is Stabilized by Ala-1 and Pro-68. The structural water is absent in the two crystal forms of the triple mutant as well as in D99N (Kumar et al., 1994). In both triple mutant crystal forms, the mutation has moved Ala- 1 markedly toward the void created by the absent structural water while Pro-68 has moved less than Ala-1. As in the D99N mutant, in the orthorhombic triple mutant, the carbonyl oxygen of Ala-1 makes a direct hydrogen bond with $\mathrm{N} \delta 2$ of the Asn-99, which is not the case in the trigonal triple mutant. The crystallographic results suggest that Ala-1 and Pro-68 are crucial for preserving the active site conformation after expelling the conserved structural water. In both the $\mathrm{D} 99 \mathrm{~N}$ and triple mutants, it appears that the hydrogen bond link between the catalytic site (D99-H48) and the $\mathrm{N}$-terminal region is retained.

The Interfacial Activation Is Only Minimally Affected by the D99N Mutation. The kinetic and equilibrium binding results with D99 mutants of PLA2 show that this residue plays a critical role in the chemical step of the catalytic turnover cycle. In conjunction with the results with mutants of D49 (Li et al., 1994), where the free carboxyl group provides two ligands for the binding of the catalytic calcium, it may be concluded that the D99 is important for the chemical step of the interfacial catalytic turnover cycle by PLA2. This conclusion is in general accord with the current consensus for the catalytic mechanism in which the H48D99 diad is important for the chemical step of PLA2 (Verheij et al., 1980; Scott et al., 1991a). 
Our results also provide insight on a few issues that could not be addressed before: that the H48-D99 diad is not required for the binding of the enzyme to the interface and that, while the binding of substrate mimics to the active site of the enzyme at the interface requires calcium ( $\mathrm{Yu}$ et al., 1993), the D99 residue is not critical for the binding of the active site-directed ligands including calcium and the substrate mimics. It may also be concluded that the $K_{\mathrm{S}}$ type of interfacial allosteric activation (Jain et al., 1993) or the micelle- or salt-induced apparent "interfacial activation" mechanisms (DeHaas et al., 1971; Verheij et al., 1981) are not noticeably altered in the D99N mutants. In short, these and other studies (Jain et al., 1995) clearly establish the role of the H48-D99 diad in the chemical step of the catalytic cycle. This provides a suitable starting point for elaborating additional details of the catalytic mechanism of PLA2.

The structural basis for the catalytic behavior of D99N may be found in the X-ray crystal structure of the D99N (Kumar et al., 1994) and the triple mutant in both forms. In the wild-type enzyme the anti $(\delta 2)$ oxygen of D99 is hydrogen bonded to $\epsilon \mathrm{NH}$ of H48. In D99N mutants the carbonyl group of the N99 side chain is hydrogen bonded to $\mathrm{H} 48$ in the same way, thus retaining the tautomeric form of $\mathrm{H} 48$ and activity. This in part may explain the differences in catalytic aspartate substitutions in PLA2 versus trypsin [see also Kuipers et al. (1990), Kumar et al. (1994), and Annand et al. (1996)]. In trypsin the syn oxygen of the catalytic aspartate is hydrogen bonded to the histidine; thus a mutation of the aspartate to asparagine would have a more pronounced effect on the interactions between the aspartate and the histidine, which would lower the catalytic activity to a much higher degree.

Role of the Hydrogen-Bonding Network in Catalytic Turnover. D99N substitution has a significant effect on the hydrogen-bonding network seen in the native structure of PLA2 (Dijkstra et al., 1981a,b). That the hydrogen bonding of Y73 to the syn $(\delta 1)$ oxygen of D99 is not critical for the catalysis is shown by the virtually unaltered catalytic behavior of the Y73F mutant (Dupureur et al., 1992a). The anti $(\delta 2)$ oxygen of Asn-99 is apparently not capable of supporting the hydrogen bond network through Y52, conserved water, and Ala-1 (Figure 1). In fact, the conserved structural water is absent in the D99N and triple mutant structure where a direct hydrogen bond is present between the carbonyl group of Ala-1 and the $\mathrm{NH}_{2}$ group of Asp-99. Since all the activity is retained in the Y52,73F mutant and significant catalytic activity is retained in the D99N and D99N/Y52,73F mutants, these results further support our earlier conclusion (Kumar et al., 1994; Liu et al., 1995; Maliwal et al., 1994) that the normal H-bonding network (Figure 1) seen in the native structure plays only a minor role in the catalytic turnover. Since the $K_{S}$ type and the apparent interfacial activation are also seen with the D99N mutant, it may be concluded that the normal H-bonding network does not play a significant role in either type of interfacial activation. This conclusion is also consistent with the observation that the bee venom PLA2 does not contain the conserved structural water or the H-bonding network (Scott et al., 1990b).

While such considerations rule out a direct role of the $\mathrm{H}$-bonding network in the apparent interfacial activation and in the catalytic cycle, the fact remains that the perturbation of the hydrogen-bonding contacts to D99 does indeed have a significant effect on $k_{\text {cat }}$. Although the kinetics of the
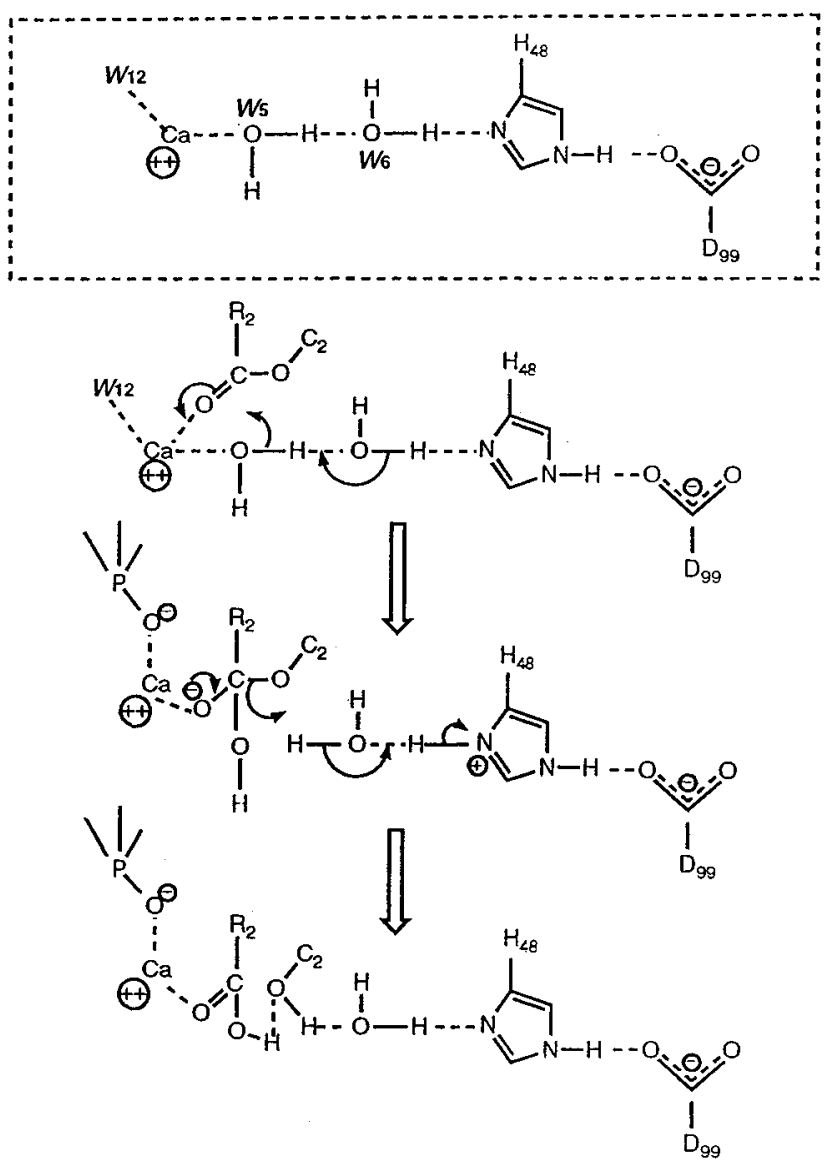

FIGURE 9: Proposal for the role of $\mathrm{Ca}$ and His-48 in the catalytic mechanism of PLA2 where the rate-limiting step is postulated to be the decomposition of the tetrahedral intermediate.

mutants are only modestly perturbed, they have been analyzed in detail. By now we have characterized several mutants in which the H-bond network (Figure 1) has been severely perturbed. In the N-terminal deletion mutants (Maliwal et al., 1994) and in the Y52V mutant (Dupureur et al., 1992b) the primary perturbation is a lower affinity for the binding of the substrate, i.e., higher $K_{\mathrm{S}} *\left(=k_{-1} / k_{1}\right)$ values. Interestingly, the D99N mutants seem to have lower $K_{\mathrm{S}}{ }^{*}$ and $K_{\mathrm{P}} *\left(=k_{3} / k_{-3}\right)$. In terms of the kinetic scheme:

$$
\mathrm{E}^{*}+\mathrm{S} \underset{k_{-1}}{\stackrel{k_{1}}{\rightleftharpoons}} \mathrm{E}^{*} \mathrm{~S} \stackrel{k_{2}}{\longrightarrow} \mathrm{E}^{*} \mathrm{P} \underset{k_{-3}}{\stackrel{k_{3}}{\rightleftharpoons}} \mathrm{E}^{*}+\mathrm{P}
$$

these results imply that in D99 mutants either the rate of decomposition of $\mathrm{E}^{*} \mathrm{~S}$ is higher or the rate of decomposition of $\mathrm{E}^{* \mathrm{P}}$ is lower. Lower $k_{3}$ corresponding to lower $K_{\mathrm{P}} *$ for the D99N mutants is also consistent with the fact that the $\mathrm{O} / \mathrm{S}$ element effect is lower; i.e., the release of products is slower compared to the formation of $\mathrm{E}^{*} \mathrm{P}$. Thus, a postulated role of the $\mathrm{H}$-bond network will be in the kinetics of the decomposition of the $\mathrm{E}^{*} \mathrm{~S}$ or $\mathrm{E}^{*} \mathrm{P}$.

Implication of O/S Ratios on the Catalytic Mechanism of PLA2. The O/S element effect for D99 mutants is significantly lower. This could imply that the nature of the chemical step or the rate-limiting step is altered in these mutants. Recently, Rogers et al. (1996) proposed an alternative mechanism of PLA2 catalysis shown in Figure 9. This mechanism differs from the current consensus mechanism for the general base catalysis by PLA2 (Verheij et al., 1980; Scott et al., 1990a) in two significant ways. First, the rate-limiting step is the decomposition of the tetrahedral intermediate. Second, two different water molecules are 
involved in the formation and the breakdown of the tetrahedral intermediate. The water involved in the formation of the tetrahedral intermediate is mediated by calcium (W5) whereas that involved in the breakdown is mediated by His48 (W6). The role of His-48 as a proton acceptor is to stabilize W6 required for the proton donation during the decomposition of the tetrahedral intermediate. That the ratelimiting step is the breakdown of the tetrahedral intermediate is consistent with the higher $\mathrm{O} / \mathrm{S}$ ratio for WT PLA2. The decrease in the $\mathrm{O} / \mathrm{S}$ ratio in the mutants suggests that the energy barrier for the formation step has been raised and becomes rate-limiting in the mutants. Thus the rate-limiting step in D99N mutants would be the formation of the tetrahedral intermediate, presumably because stabilization of protonated W6 with $\mathrm{H} 48$ is perturbed due to lack of coupling with anionic D99.

Conclusion. In summary, X-ray evidence clearly demonstrates that in both the D99N and triple mutant the hydrogen-bonding network which links the catalytic site to the interfacial binding region is altered and the structural water is excluded. The amino group of the amide residue of the mutant Asn-99 pushes out the structural water and directly hydrogen bonds to the carbonyl oxygen of Ala-1, resulting in its movement. It appears that the amino group is needed to push the water out. However, a modified hydrogen bond link is maintained, and the catalytic machinery including $\mathrm{H} 48$, calcium, and the three water molecules (W5, W6, and W12) is not measurably perturbed. Furthermore, the normal hydrogen-bonding network involving the structural water present in the native enzyme is not crucial for any form of interfacial activation, and the effect on catalysis is modest at best. The D99N and triple mutants with the modified hydrogen-bonding connections still possess reduced catalytic activity. Detailed kinetic analysis suggested that the kinetic effect primarily lies in $k_{\text {cat }}$ and that the ratelimiting step has possibly been altered in the mutants.

\section{REFERENCES}

Annand, R. R., Kontoyianni, M., Penzoth, J. E., Dudler, T., Leybrand, T. P., \& Gelb, M. H. (1996) Biochemistry 35, 45914601.

Berg, O. G., Yu, B.-Z., Rogers, J., \& Jain, M. K. (1991) Biochemistry 30, 7283-7297.

Brunger, A. T. (1992) X-PLOR Manual, Yale University, New Haven, CT.

Cajal, Y., Rogers, J., Berg, O. G., \& Jain, M. K. (1996) Biochemistry 35, 299-308.

DeHaas, G. H., Bonsen, P. P. M., Pieterson, W. A., \& Van Seenen, L. L. M. (1971) Biochim. Biophys. Acta 239, 252-266.

Dijkstra, B. W., Drenth, J., \& Kalk, K. H. (1981a) Nature 289, 604-606.

Dijkstra, B. W., Kalk, K. H., Hol, W. G. J., \& Drenth, J. (1981b) J. Mol. Biol. 147, 97-123.

Dijkstra, B. W., Renetseder, R., Kalk, K. H., Hol, W. G. J., \& Drenth, J. (1983) J. Mol. Biol. 1681, 63-179.

Dupureur, C. M., Yu, B.-Z., Jain, M. K., Noel, J. P., Deng, T., Li, Y., Byeon, I. L., \& Tsai, M.-D. (1992a) Biochemistry 31, 64026413.

Dupureur, C. M., Yu, B.-Z., Memone, J. A., Jain, M. K., \& Tsai, M.-D. (1992b) Biochemistry 31, 10576-10583.

Huang, B., Yu, B.-Z., Rogers, J., Byeon, I.-J. L., Sekar, K., Chen, K., Sundaralingam, M., Jain, M. K., \& Tsai, M.-D. (1996) Biochemistry 35, 12164-12174.

Jain, M. K., \& Vaz, W. L. C. (1987) Biochim. Biophys. Acta 905, $1-8$.

Jain, M. K., \& Maliwal, B. P. (1993) Biochemistry 32, 1183811846.

Jain, M. K., Egmond, M. R., Verheij, H. M., Apitz-Castro, R. J., Dijkman, R., \& de Haas, G. H. (1982) Biochim. Biophys. Acta $688,341-348$.
Jain, M. K., Rogers, J., Jahagirdar, D. V., Marecek, J. F., \& Ramirez, F. (1986a) Biochim. Biophys. Acta 860, 435-447.

Jain, M. K., Maliwal, B. P., de Haas, G. H., \& Slotboom, A. J. (1986b) Biochim. Biophys. Acta 860, 448-461.

Jain, M. K., Rogers, J., Marecek, J. F., Ramirez, F., \& Eibl, H. (1986c) Biochim. Biophys. Acta 860, 462-474.

Jain, M. K., Rogers, J., \& de Haas, G. H. (1988) Biochim. Biophys. Acta 940, 51-62.

Jain, M. K., Yu, B.-Z., Rogers, J., Ranadive, G. N., \& Berg, O. G. (1991a) Biochemistry 30, 7306-7317.

Jain, M. K., Rogers, J., Berg, O., \& Gelb, M. H. (1991b) Biochemistry 30, 7340-7348.

Jain, M. K., Tao, W., Rogers, J., Arenson, C., Eibl, H., \& Yu, B.Z. (1991c) Biochemistry 30, 10256-10268.

Jain, M. K., Yu, B.-Z., \& Berg, O. G. (1993) Biochemistry 32, 11319-11329.

Jain, M. K., Gelb, M. H., Rogers, J., \& Berg, O. G. (1995) Methods Enzymol. 249, 567-614.

Jain, M. K., Rogers, J., Yu, B.-Z., Yao, J., \& Romsted, L. S. (1997) (to be submitted for publication).

Jones, T. A. (1985) Methods Enzymol. 115, 157-171.

Kuipers, O. P., Franken, P. A., Hendricks, R., Verheij, H. M., \& de Haas, G. H. (1990) Protein Eng. 4, 199-204.

Kuipers, O. P., Vincent, M., Brochon, J., Verheij, H. M., de Haas, G. H., \& Gallay, J. (1991) Biochemistry 30, 8771-8785.

Kumar, A., Sekharudu, Y. C., Ramakrishnan, B., Dupureur, C. M., Zhu, H., Tsai, M.-D., \& Sundaralingam, M. (1994) Protein Sci. 3, 2082-2088.

Laskowski, R. A., MacArthur, M. W., Moss, D. S., \& Thornton, J. M. (1993) J. Appl. Crystallogr. 26, 283-291.

Li, Y., \& Tsai, M.-D. (1993) J. Am. Chem. Soc. 115, 8523-8326.

Li, Y., Yu, B.-Z., Zhu, H., Jain, M. K., \& Tsai, M.-D. (1994) Biochemistry 33, 14714-14722.

Liu, X., Zhu, H., Huang, B., Yu, B.-Z., Rogers, J., Kumar, A., Jain, M. K., Sundaralingam, M., \& Tsai, M.-D. (1995) Biochemistry 34, 7322-7334.

Luzzati, V. (1952) Acta Crystallogr. 5, 802-807.

Maliwal, B. P., Yu, B. Z., Szacinski, H., Squier, T., van Binsbergen, J., Slotboom, A. J., \& Jain, M. K. (1994) Biochemistry 33, 45094516.

Noel, J. P., Bingman, C., Deng, T., Dupureur, C. M., Hamilton, K. J., Jiang, R.-T., Kwak, J.-G., Sekharudu, Y. C., Sundaralingam, M., \& Tsai, M.-D. (1991) Biochemistry 30, 11801-11811.

Pieterson, W. A., Vidal, J. C., Volwerk, J. J., \& de Haas, G. H. (1974) Biochemistry 13, 1455-1460.

Ramirez, F., \& Jain, M. K. (1991) Proteins 9, 229-239.

Rogers, J., Yu, B. Z., Serves, S. V., Tsivgoulis, G. M., Sotivopoulos, D. N., Ioannou, P. V., \& Jain, M. K. (1996) Biochemistry 35, 9375-9385.

Scott, D. L., White, S. P., Otwinowski, Z., Yuan, W., Gelb, M. H., \& Sigler, P. B. (1990a) Science 250, 1541-1546.

Scott, D. L., Otwinowsi, Z., Gelb, M. H., \& Sigler, P. B. (1990b) Science 250, 1563-1566.

Sekharudu, Y. C., Ramakrishnan, B., Huang, B., Jiang, R.-T., Dupureur, C. M., Tsai, M.-D., \& Sundaralingam, M. (1992) Protein Sci. 1, 1585-1594.

Seshadri, K., Vishveshwara, S., \& Jain, M. K. (1994) Proc. Indian Acad. Sci. 106, 1177-1189.

Thunnissen, M. M. G. M., Ab, E., Kalk, K. H., Drenth, J., Dijkstra, B. W., Kuipers, O. P., Dijkman, R., de Haas, G. H., \& Verheij, H. M. (1990) Nature 347, 689-691.

Van den Berg, B., Tessari, M., Boelens, R., Dijkman, R., Kaptein, R., de Haas, G. H., \& Verheij, H. M. (1995) J. Biomol. NMR 5 , $110-121$.

Verheij, H. M., Slotboom, A. J., \& de Haas, G. H. (1981) Rev. Physiol. Biochem. Pharmacol. 91, 91-203.

Volwerk, J. J., \& de Haas, G. H. (1982) in Lipid Protein Interactions (Jost, P. C., \& Griffith, O. H., Eds.) Vol. 1, pp 69-149, John Wiley \& Sons, New York.

Weis, W. I., Drikamer, K., \& Hendrickson, W. A. (1992) Nature $360,127-134$.

Yu, B.-Z., Berg, O. G., \& Jain, M. K. (1993) Biochemistry 32, 6485-6492.

BI961576X 